\title{
Self-similar solutions of laser produced blast waves
}

\author{
K P J REDDY \\ Department of Aerospace Engineering, Indian Institute of Science, Bangalore 560012, India
}

MS received 3 May 1995; revised 27 July 1995

\begin{abstract}
The aerodynamics of the blast wave produced by laser ablation is studied using the piston analogy. The unsteady one-dimensional gasdynamic equations governing the flow are solved under assumption of self-similarity. The solutions are utilized to obtain analytical expressions for the velocity, density, pressure and temperature distributions. The results predict all the experimentally observed features of the laser produced blast waves.
\end{abstract}

Keywords. Ablation; laser; blast wave; shock wave; gasdynamics.

PACS Nos $42 \cdot 60 ; 52 \cdot 50 ; 36 \cdot 20 ; 61 \cdot 80$

\section{Introduction}

The laser ablation is a dry photoetching technique with submicron feature resolution [1] and ability to remove material in submicron layers [2]. This technique is useful in photoetching of polymers for lithographic applications [3] and biological tissues as in laser surgery [4]. Recent studies on the laser ablation of biological tissues and synthetic polymers like polymethyl methacrylate(PMMA), polyimide, and polyethylene-terephthalate (PET) has led to the new phenomenon called ablative photodecomposition which results in the ejection of the ablated material at supersonic velocities [5]. These studies have addressed various aspects of laser surface ablation processes such as identifying the emission products in the plume through spectroscopic means [6], the measurement of their expansion velocities through ultrafast microscopy [7] and self-focusing of the laser pulse in the medium and delay in the appearance of the gas phase products above the surface [8].

In vacuum, due to the absence of any resistance from the surrounding gas, the ejected target material particles disperse ballistically at Mach numbers $\sim 150$. While in presence of the background gas, such as air, nitrogen, oxygen and argon, the ejected material travels at Mach numbers $\sim 20$ and produces a strong spherical shock wave which expands into the surrounding ambient gas engulfing more and more background gas. When the mass $m_{0}$ of this shocked gas exceeds that of the ablated gas $m_{\mathrm{a}}$ the shock wave develops into a blast wave. Since the energy contained in the laser pulse is finite, the expansion velocity of the shock front decreases with increasing time. Most of the studies reported are the experimental investigations of the laser ablation process and the spectroscopic analysis of the plumes produced due to ablation. However, very few theoretical studies have been reported on the propagation dynamics of the ejected material from the sample surface. It is important to analyze the processes of expansion 
of the ejected material and to study the variation of pressure, density and temperature as the material expands above the target surface. The aim of this paper is to present the theoretical analysis of the expansion dynamics of the blast wave generated by the material ejected due to laser ablation. Strong spherical blast wave created under the assumption $m_{0} \gg m_{\mathrm{a}}$ is considered in the present analysis. This analysis uses the piston analogy where the flow is governed by a set of unsteady one-dimensional gasdynamic equations which are solved using the self-similar solutions method $[9,10]$. The solutions are used to get analytical expressions for the pressure, density and the temperature distributions along the radial direction of the spherical blast wave produced by laser ablation starting from the centre of explosion. The analysis of cylindrical blast waves where $m_{0} \leqslant m_{\mathbf{a}}$ has been presented recently [11].

\section{Governing equations}

In an idealized situation the problem of the blast wave generated by the irradiation of a polymer surface or a biological tissue with ultrashot laser pulse of high energy in presence of ambient gas is equivalent to an explosion at time $t=0$ in a gas at rest at the centre of symmetry with an instant liberation of finite amount of energy $E_{0}$ (which is a sum of kinetic energy and the heat energy), given by

$$
E_{0}=\int_{0}^{R}\left(\frac{1}{2} \rho u^{2}+\frac{1}{\gamma-1} p\right) r^{2} \mathrm{~d} r
$$

where $u, \rho$, and $p$ are the velocity, density and the pressure of the gas in the blast wave, $r$ is the radial coordinate and $\gamma$ is the specific heat ratio of the ambient gas.

The ejected material due to ablation acts as a piston which drives a spherical blast wave into the ambient gas whose radius $R$ increases with the increasing time as

$$
R=\left(\frac{E}{\rho_{0}}\right)^{1 / 5} t^{2 / 5}
$$

More and more background gas gets swept by the shock front at the leading edge of the blast wave which travels at a velocity $U$ defined by

$$
U=\frac{\mathrm{d} R}{\mathrm{~d} t}=\frac{2}{5} \sqrt{\frac{\mathrm{E}}{\rho_{0}}} \frac{1}{\sqrt{R^{3}}}
$$

where, $E$ is a constant related to the energy $E_{0}$ through the relation $E_{0}=\alpha(\gamma) E$ in which $\alpha(\gamma)=0.851$ for air or nitrogen [9].

The energy $E_{0}$ of the blast wave defined in (1) can be determined from the experimentally measured radius of the expanding spherical blast wave for the known values of the incident laser pulse by rewriting (2) in the following form

$$
\frac{5}{2} \log R-\log t=\frac{1}{2} \log \left(\frac{E}{\rho_{0}}\right) .
$$

For the narration of the usefulness of the above equation we replot the data given in [12] in the form shown in figure 1. This data has been obtained using a laser pulse of energy $370 \mu \mathrm{j}$ (incident power of $54 \mathrm{GW} / \mathrm{cm}^{2}$ ) at $532 \mathrm{~nm}$ to generate the blast wave from 


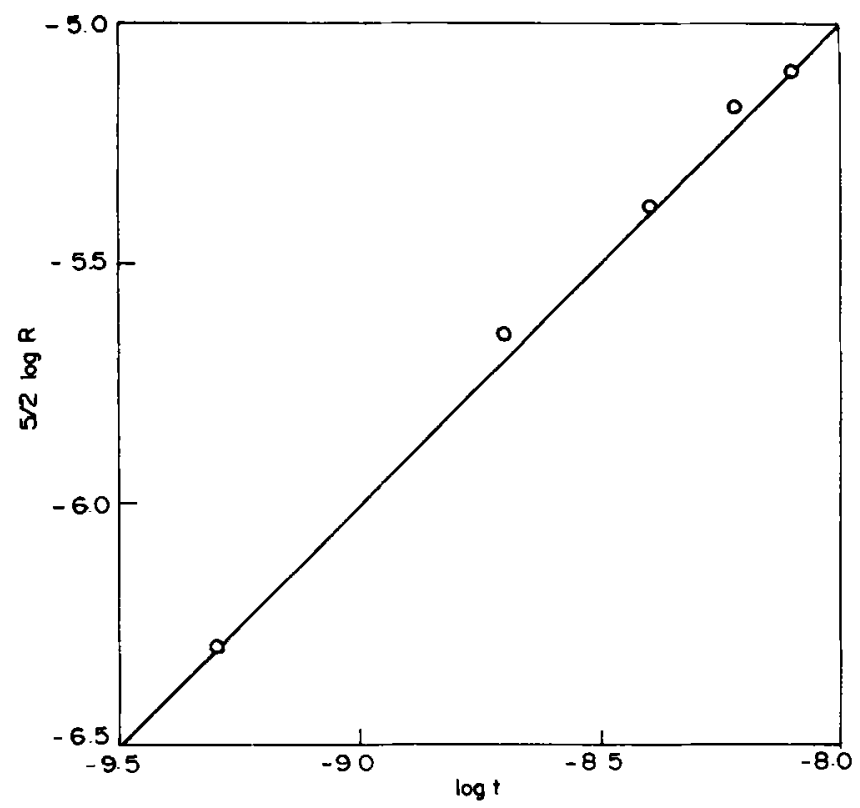

Figure 1. Experimentally measured radius $R(\mathrm{~cm})$ of the laser produced blast wave as a function of time (sec) (data replotted from [12]).

the surface of a PMMA. The laser pulse is generated by frequency doubling the output from a regenerative amplifier which amplifies 80 ps duration pulses from a mode locked $\mathrm{Nd}$ :YAG laser at $1.06 \mu \mathrm{m}$. This pulse is divided into damage and probe pulses and focused on to the sample surface in a vacuum chamber. The probe pulse with flux $<1 \%$ of the damage pulse is imaged by a camera with diffraction-limited imaging ability. The experimental data agrees with the theoretical formula given by (4) if we assume $1 / 2 \log \left(E / \rho_{0}\right)=2.993$, which yields $E=163.86 \mu \mathrm{j}$. Assuming an ambient air pressure of $1 \mathrm{~atm}$. and temperature of $298 \mathrm{~K}$ this yields the blast wave energy $E_{0}=139.446 \mu \mathrm{j}$. This energy agrees with the blast wave energy estimated earlier [12].

The motion of the gas in the blast wave is governed by the following set of unsteady gasdynamic equations [13],

$$
\begin{aligned}
& \frac{\partial u}{\partial t}+u \frac{\partial u}{\partial r}+\frac{1}{\rho} \frac{\partial p}{\partial r}=0 \\
& \frac{\partial \rho}{\partial t}+u \frac{\partial \rho}{\partial r}+\rho \frac{\partial u}{\partial r}+\rho \frac{2 u}{r}=0 \\
& \frac{\partial}{\partial t}\left(\frac{p}{\rho^{\gamma}}\right)+u \frac{\partial}{\partial r}\left(\frac{p}{\rho^{\gamma}}\right)=0
\end{aligned}
$$

along with the equation of state $p=\rho R T$. Equations (5) to (7) are the momentum, continuity and energy equations, respectively. 
The aim of the present analysis is to solve the above set of equations and obtain analytic expressions for the distributions of the flow variables along the radial coordinate $r$ starting from $r=0$ at the centre of the explosion.

\section{Self-similar solutions}

It is well-known that an abrupt jump in the flow parameters takes place on the boundaries where the perturbed gas due to the blast wave is separated from the unperturbed gas by a shock front of radius $R$. These jump conditions given by the Rankine-Hugoniot conditions, define the velocity, density and the pressure behind the shock front as

$$
\begin{aligned}
& u_{2}=\frac{2 U}{\gamma+1} f_{1} \\
& \rho_{2}=\frac{\gamma+1 \rho_{0}}{\gamma-1} f_{2} \\
& p_{2}=\frac{2 U^{2} \rho_{0}}{\gamma+1} f_{3}
\end{aligned}
$$

where, $\rho_{0}$ is the density of the undisturbed gas, $f_{1}=1-a^{2} / U^{2}, f_{2}=\left(1+2 a^{2} /\right.$ $\left.U^{2}(\gamma-1)\right)^{-1}, f_{3}=1-a^{2}(\gamma-1) /\left(2 \gamma U^{2}\right)$, and $a$ is the speed of sound in the medium.

In the laser produced blast wave it is shown that the pressure behind the shock front is very high compared to the pressure ahead of the shock wave [7]. The pressure ahead of the shock wave can be neglected in comparison with the pressure behind the shock wave. However, it is important to estimate with what accuracy and for which shock waves this assumption is valid. The values of $f_{1}, f_{2}$, and $f_{3}$ in the above equations differ from unity by $<5 \%$ when the ratio $a / U<0 \cdot 1$ (that is for the stronger shocks). Thus if we use $a / U=0$ and $f_{1}=f_{2}=f_{3}=1$, which is same as assuming counter pressure $p_{0}=0$, then an error of $<5 \%$ is introduced into the values of $u, \rho$, and $p$. Then the Rankine-Hugoniot conditions reduce to the following form after substituting $U$ from (3),

$$
\begin{aligned}
& u_{2}=\frac{4}{5(\gamma+1)}\left(\frac{E}{\rho_{0}}\right)^{1 / 2} R^{-3 / 2} \\
& \rho_{2}=\frac{\gamma+1}{\gamma-1} \rho_{0} \\
& p_{2}=\frac{8 E}{25(\gamma+1)} R^{-3} .
\end{aligned}
$$

The corresponding temperature is determined using the equation of state.

The system of characteristic parameters influencing the motion of the perturbed gas after the explosion, governed by (5)-(7), under adiabatic conditions is represented by the quantities $p_{0}, \rho_{0}, E_{0}, r, t$ and $\gamma$. By non-dimensionalizing the basic equations we can show that the non-dimensional variables depend only on the dimensionless parameters $\gamma, \lambda=\rho_{0}^{1 / 5} r / E_{0}^{1 / 5} t^{2 / 5}$ and $\tau=p_{0}^{5 / 6} t / E_{0}^{1 / 3} \rho_{0}^{1 / 2}$, of which $\lambda$ and $\tau$ are variables. By neglecting the counterpressure the variable $\tau$ disappears. In this case the flow variables change with time in a manner that their distributions with respect to the coordinate variable 


\section{Laser produced blast waves}

$r$ always remain similar in time. Then such a flow can be considered as self-similar. However, as the shock wave travels away from the centre of explosion it becomes weaker and the pressure on either side of the shock front become comparable. Therefore in such a situation the counterpressure cannot be neglected and hence the flow ceases to be self-similar.

The self-similar solution for the laser produced blast wave can be expressed in terms of the non-dimensional velocity $V$, which varies in the range $2 / 5 \gamma \leqslant V \leqslant 4 / 5(\gamma+1)$ where, $V=4 / 5(\gamma+1)$ corresponds to the shock front, and the value $V=2 / 5 \gamma$ corresponds to the centre of the explosion. The complete solutions normalized with respect to the values behind the shock front are [9],

$$
\begin{aligned}
\frac{r}{R}= & {\left[\frac{5}{4}(\gamma+1) V\right]^{-2 / 5}\left[\frac{(\gamma+1)}{(\gamma-1)}\left(\frac{5}{2} \gamma V-1\right)\right]^{-\alpha_{2}} } \\
& \times\left[\frac{5(\gamma+1)}{5(\gamma+1)-2[2+3(\gamma-1)]}\left(1-\frac{2+3(\gamma-1)}{2} V\right)\right]^{-\alpha_{3}} \\
\frac{u}{u_{2}}= & \frac{5}{4}(\gamma+1) \frac{r}{R} V \\
\frac{\rho}{\rho_{2}}= & {\left[\frac{(\gamma+1)}{(\gamma-1)}\left(\frac{5}{2} \gamma V-1\right)\right]^{\alpha_{3}}\left[\frac{(\gamma+1)}{(\gamma-1)}\left(1-\frac{5}{2} V\right)\right]^{\alpha_{3}} } \\
\frac{p}{p_{2}}= & {\left[\frac{5}{4}(\gamma+1) V\right]^{6 / 5}\left[\frac{(\gamma+1)}{(\gamma-1)}\left(1-\frac{5}{2} V\right)\right]^{\alpha(\gamma+1)} } \\
& \times\left[\frac{5(\gamma+1)}{5(\gamma+1)-2[2+3(\gamma-1)]}\left(1-\frac{2+3(\gamma-1)}{2} V\right)\right]^{\alpha_{4}} \\
\frac{T}{T_{2}}= & \frac{p}{p_{2}} \frac{\rho_{2}}{\rho}
\end{aligned}
$$

where, $\alpha_{1}=1.4565, \alpha_{2}=-0.563, \alpha_{3}=0.78947, \alpha_{4}=12.1375$ and $\alpha_{5}=-3.333$ if we assume air or nitrogen as the ambient gas surrounding the target $(\gamma=1.4)$.

The distribution of velocity, density, pressure and the temperature behind the shock front along the radial coordinate $r$ computed from the above equations are shown in figure 2. These results show that the velocity and density tend to zero near the centre of symmetry while the pressure reaches a constant value and the temperature tends to infinity. Thus large temperature gradients occur and the mass of the gas disperses near the centre of symmetry where the explosion takes place. The pressure is finite at the centre but decays to zero as time increases. Hence a reverse gas motion towards the centre of ablation must occur after a lapse of finite time when the etching is achieved by the irradiation with laser pulses of very high fluence. This process confines the ejected fragments of the sample closer to the ablation centre and prevents the dispersion of the fragments away from the target surface. This phenomenon has been observed in the laser ablation of $\mathrm{YBa}_{2} \mathrm{Cu}_{3} \mathrm{O}_{7-\delta}$ target [14] and other materials [6]. 


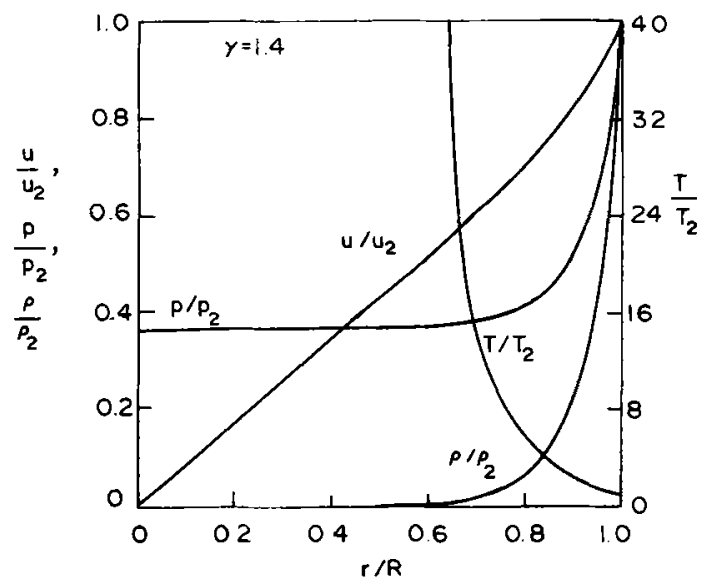

Figure 2. Distribution of the flow variables behind the shock wave in the laser produced spherical blast wave along the radial distance $r$.

For the case of very intense laser pulse the analytic asymptotic expressions for the velocity, density, pressure and temperature near the centre of the explosion can be obtained from the above equations in the limits $V \rightarrow 2 / 5 \gamma$ and $r \rightarrow 0$ as

$$
\begin{aligned}
& u=\frac{2}{5 y} \frac{r}{t} \\
& \rho=k_{1} \rho_{0}\left(\frac{E}{\rho_{0}}\right)^{-(3 / 5(\gamma-1))}(t)^{-(6 / 5(\gamma-1))}(r)^{3 /(5(\gamma-1))} \\
& p=k_{2} \rho_{0}\left(\frac{E}{\rho_{0}}\right)^{2 / 5}(t)^{-6 / 5} \\
& T=k_{3}\left(c_{\mathrm{v}}\right)^{-1}\left(\frac{E}{\rho_{0}}\right)^{(2(\gamma-1)+3) /(5(\gamma-1))}(t)^{(6(2-\gamma)) /(5(\gamma-1))}(r)^{-3 /(\gamma-1)}
\end{aligned}
$$

where, $k_{1}, k_{2}$ and $k_{3}$ are the functions of the specific heat ratio $\gamma$ and $c_{v}$ is the specific heat coefficient at constant volume.

A contact surface separates the shocked gas and the plume containing the fragments of the ablated material which drives the blast wave. Hence the value of the specific gas constant changes at the contact surface as we approach the centre of the explosion starting from the shock front. Therefore appropriate value of $\gamma$ should be used to utilize (20)-(21) to compute the flow variables. This values for the plume can be determined experimentally.

\section{Conclusions}

The blast wave phenomenon produced due to the ablative photodecomposition occurring during the photoetching of a polymer surface or a biological tissue using high 


\section{Laser produced blast waves}

fluence laser pulses is analyzed. Based on the piston analogy the unsteady onedimensional gasdynamic equations are solved under the self-similarity condition for the spherical blast wave. The similar solutions yield analytic expressions for the distribution of flow variables. Asymptotic analytic expressions for the field variables near the centre of explosion where the laser pulse interacts with the target are obtained from the general solutions. The analysis predicts the experimentally observed features of the confinement of the plume closer to the target surface in presence of the blast wave.

\section{References}

[1] D Henderson, J C White, H G Craighead and I Adesida, Appl. Phys. Lett. 46, 900 (1985)

[2] R Srinivasan and B Braren, J. Polym. Sci. 22, 2601 (1984)

[3] J T C Yeh, J. Vac. Sci. Technol. A4, 653 (1986)

[4] R J Lane, R Linsker, J J Wynne, A Torres and R G Geronemus, Arch. Dermatol. 121, 609 (1985)

J J Haller, M H Wholley, E R Fisher, E M Krokosky and R Srinivasan, Cardio 2, 31 (1985)

H Nomes, R Srinivasan, R Solanki and E Johnson, Soc. Neurosci. 11, 1167 (1985)

J Marshall, S Trokel, S Rothery and R R Krueger, Br. J. Ophthalmol. 70, 482 (1986)

[5] R Srinivasan, Science 234, 559 (1986)

[6] P E Dyer and J Sidhu, J. Appl. Phys. 64, 4657 (1988)

[7] T Zyung, H Kim, J C Postlewaite and D D Dlott, J. Appl. Phys. 65, 4548 (1989)

[8] H Kim, J C Postlewaite, T Zyung and D D Dlott, Appl. Phys. Lett. 54, 2274 (1989)

[9] L I Sedov, Similarity and dimensional methods in mechanics (Mir, Moscow, 1982)

[10] Ya B Zeldovich and Yu P Raizer, Physics of shock waves and high temperature hydrodynamic phenomena edited by W D Hayes and R F Probstein (Academic, New York. 1966) Vols. I and II

[11] G J Hutchens, J. Appl. Phys. 77, 2912 (1995)

[12] H Kim, J C Postlewaite, T Zyung and D D Dlott, J. Appl. Phys. 64, 2955 (1988)

[13] A Sakurai, J. Phys. Soc. Jpn. 8, 662 (1953)

[14] A Gupta, B Braren, K G Casey, B W Hussey and R Kelly, Appl. Phys. Lett. 59, 1302 (1991) 